\title{
In-Home PLC Coexistence with Wi-Fi and Ethernet access Networks
}

\author{
E. Hamiti, T. Berisha and F. Peci
}

\begin{abstract}
BPL or PLC is relatively a new technology that is considered an attractive system to deliver broadband communications over power lines. The main objective of paper is to understand the impact of in-home PLC devices in case of integrated environment with other technologies. Initially, FTW Simulator for channel transfer function analysis of an in-home PLC network topology is used. Furthermore, through measurements conducted in laboratory environment different scenarios are built for different test scenarios and the coexistence of in-home PLC, Ethernet and Wi-Fi Networks is investigated. Toward the end of the paper, throughout different scenarios the impact on video quality of in-home PLC, will be closely monitored and evaluated.
\end{abstract}

Index Terms-BPL, channel transfer function, FTW simulator, in-home PLC.

\section{INTRODUCTION}

$\mathrm{T}_{\mathrm{p}}^{\mathrm{r}}$ HE PLC (Power-line Communications) in general is a promising solution for first mile and last mile broadband access. It appears that broadband access is a broader concept related to the growth of science, economy aspects and also tourism and culture. In-home PLC, Home-plug AV and indoor BPL (Broadband over Power-lines) are dedicated standards providing a broadband access solution to in-home users. Nowadays there are a lot of PLC devices with different characteristics of transmission speeds and capabilities. Moreover Home-plug and BPL devices include products that have flown from different standards.

In this section the simulations derived from FTW are described and a simulator is used for modeling channel transfer function for in-home PLC [1,2] (FTW is PLC Simulator based on Matlab source code which is used to characterize PLC channels). Through this simulator are conducted a considerable number of simulations for different kind of topologies and characteristics. In powerline communication network the signals propagate

E. Hamiti University of Prishtina, Faculty of Electrical and Computer Engineering, Prishtina, Kosovo, Corresponding author, (email: enver.hamiti@uni-pr.edu)

T. Berisha, University of Prishtina, Faculty of Electrical and Computer Engineering, Prishtina, Kosovo, (email: taulant.berisha@uni-pr.edu)

F. Peci, University of Prishtina, Faculty of Electrical and Computer Engineering, Prishtina, Kosovo, (email: fatos.peci@uni-pr.edu) from transmitter to receiver facing a harsh and noisy transmission medium. Using the FTW simulator is possible to derive the best possible frequency band where the voice and data can be transmitted.

The simulations are performed in the frequency band from $100 \mathrm{KHz}$ up to $30 \mathrm{MHz}$ matched to the modeled characteristics of the simulator.

There are a number of basic conducted measurements performed in various scenarios as well. Measurements include different topologies and each of them designed for various purposes. By using various scenarios the importance of PLC is investigated as a redundant link during intermittent drops of one healthy link while video-streaming. In the rest of scenarios the focus is on load-sharing of packets where the topology includes two links for transmitting the signals from transmitter to receiver. The topology is composed of different technologies like Wi-Fi and Ethernet along with inhome PLC devices.

The motivation for building these kind of topologies is derived from the possibilities of using the in-home PLC not only for home use where the inside users can have access to broadband, but also in the environments like mining industry and other kind of industries where the broadband access is necessary even for monitoring purposes. The efficient bandwidth utilization is another target analyzed in measurements conducted on this paper.

The paper is organized as follows: Section II elaborates the channel transfer function $(\mathrm{H})$ for a predefined in-home PLC topology, Section III analyses the laboratory measurements and Section IV gives a conclusion.

\section{CHANNEL TRANSFER FUNCTION ANALYSIS THROUGH SIMULATIONS}

The proposed simulations in this paper include the inhome PLC network topology that matches as close as possible to a realistic indoor power-line networks in our country (Kosovo, South Eastern Europe). On the other hand the channel transfer function is defined from the theory of two-port network model as a ratio between load voltage VL and source voltage VS by:

$$
\boldsymbol{H}=\frac{V_{L}}{V_{S}}
$$

The above can be rewritten as a function of A, B, C, D, ZL, and $\mathrm{ZS}$ as follows: 


$$
H=20 X \log _{10}\left|\frac{Z_{L}}{A X Z_{L}+B+Z_{S} X\left(C X Z_{L}+D\right)}\right|
$$

where $\mathrm{A}, \mathrm{B}, \mathrm{C}, \mathrm{D}$ are the $2 \times 2$ matrix elements according to the ABCD Line Modeling, whereas ZS and ZL are the source impedance and load impedance respectively [3-6].
In this paper the in-home PLC network topology that is matched to our country environments will be presented. Referring to the topology as depicted below (Figure 1), there are a service panel (SP) positioned in the center of topology and outlets (OL) connected to the distribution boxes (DB) etc. The DBs are considered boxes positioned per each room for in-home environments. The SP connects the energy provider side to in-home electrical side.

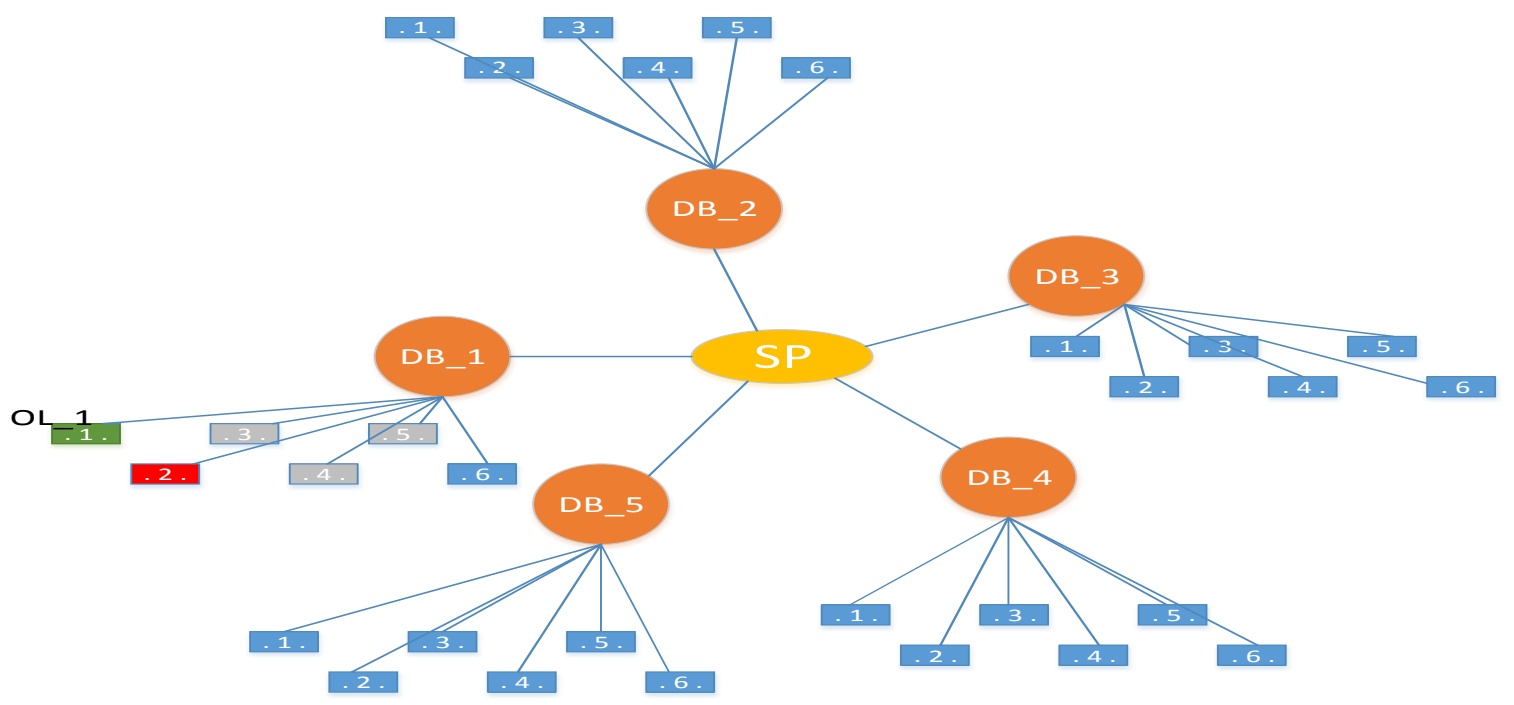

Fig.1. The topology of in-home PLC network for channel transfer function analysis

From the above topology we can see the connections between topology elements. The distance between SP and DBs is $6 \mathrm{~m}$ and is the same for five connections, whereas the distance between each DB and OLs connected to it are 5.2, 5.4, 5.6, 5.8, 6.0 and $7 \mathrm{~m}$ respectively. Cable types used in topology are of sections $2.5 \mathrm{~mm}$. Transmitter (equivalent as ZS and colored as green) and receiver (equivalent as $\mathrm{ZL}$ and colored as red) have a common value of $100 \Omega$. Star connection type is used for connection between OLs and respective DBs.

It is important to see what happens to the channel transfer function for the topology as above on situation where position of receiver changes from OL_2 to OL_3 and so on. Below are depicted the graphic results which will be analyzed on the following.

Figure 2 shows an example from which may be observed that differences between channel transfer functions are slightly change from each other.

The analysis as it can be seen from the legend is performed for the cases where receiver changes the position from one outlet to another within distribution box (DB_1 in this case) in which is connected to. If the range from transmitter $(\mathrm{T})$ to receiver $(\mathrm{R})$ is increased there is a longer path linking $\mathrm{T}$ and $\mathrm{R}$. It is expected to be corrupted the channel performance caused by this increasing. The simulations are conducted from $100 \mathrm{KHz}$ up to $30 \mathrm{MHz}$ as it can be seen from Fig. 2. Also the bandwidth from 7-15 MHz is not suitable for transmission because of high level of attenuation. Apart from transmitter and receiver the topology presented consists of RLC circuits as well. These circuits are considered as appliance circuits when are connected to outlets in randomly way. In the topologies where there is no RLC circuit connected to outlets, the bandwidth up to $5 \mathrm{MHz}$ shows an improvement of attenuation, whereas the bandwidth from 7-15 MHz remains problematic. The receivers from different vendors have their specifications related to the receiving capabilities. The power noise level is not desirable to be lower than around $-90 \mathrm{~dB}$ for getting the better performance for receiver devices. The careful should be taken mainly in the bandwidths mentioned above although nowadays devices use bandwidth up to $62.5 \mathrm{MHz}$ for networking, HD videostreaming, online gaming and more services. 


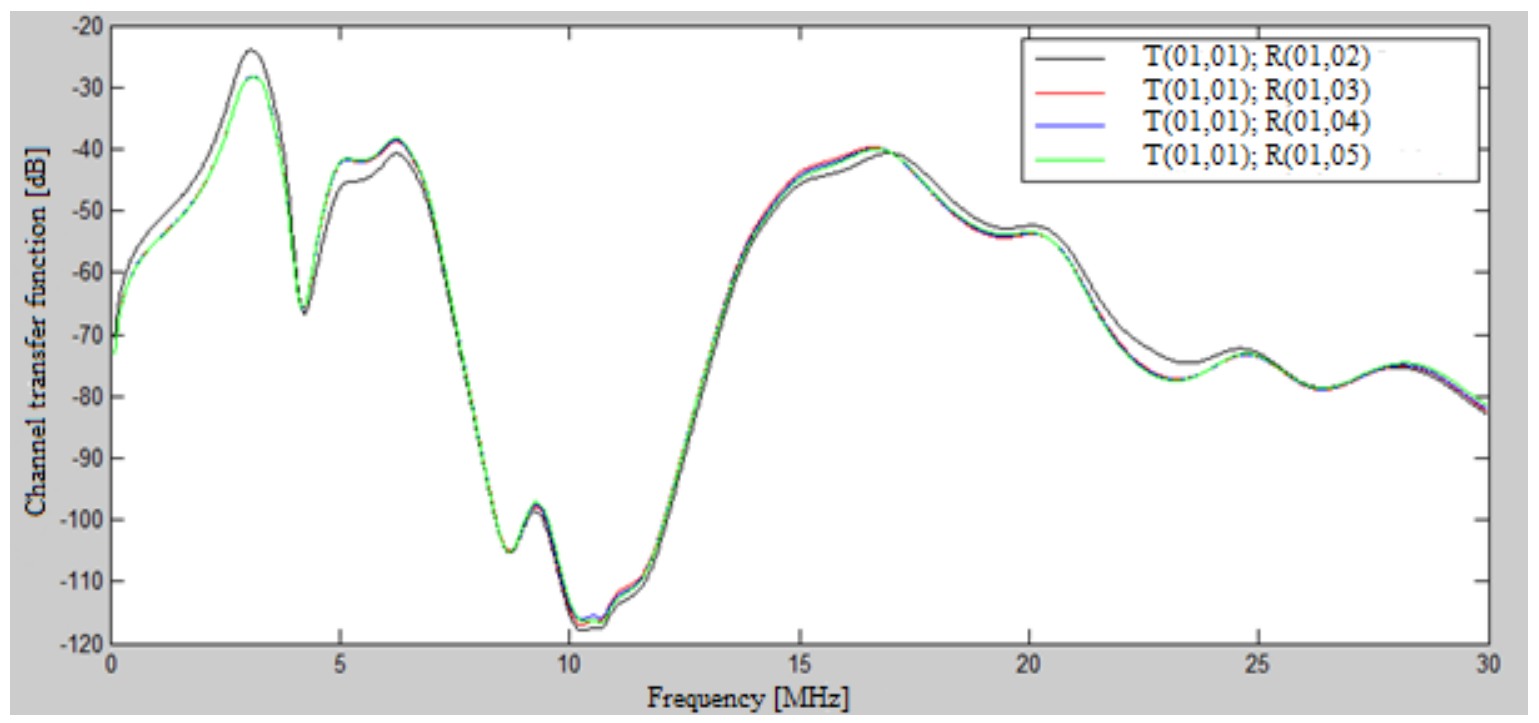

Fig.2. Channel transfer function $(\mathrm{H})$ realizations of 4 different receiver positions

\section{LABORATORY TESTBED AND IN- HOME PLC MEASUREMENTS}

Using PLC adapters for different environments when there is no need for building infrastructure is optimal solution and even cost-effective. The general purpose of measurements is to investigate the coexistence between technologies like in-home PLC, Wi-Fi and Ethernet. The jitter and latency for videostreaming and similar services like monitoring are to be analyzed whereby the results are shown on the following.

\subsection{Laboratory Test-bed}

The proposed test-bed in this paper consists of:

- 2 PLC adapters compliant with IEEE1901 and compatible with Homeplug AV [4],

- 2 PCs equipped with 100 and $1000 \mathrm{Mbps}$ NIC (Network Interface Cards),

- 2 Cisco routers

- 1 Cisco Ethernet switch (not presented on the following topologies).

Within this paper three measurement scenarios were conducted:

- Scenario 1: Bottleneck analysis near $100 \mathrm{Mbps}$ and importance of different transmission mediums.

- Scenario 2: High Redundancy analysis in case where the primary link is down (Ethernet link) and video-streaming quality between PC_1 (transmitter) and PC_2 (receiver).

- Scenario 3: Load-sharing per packet (utilizing two links, PLC in combination with Wi-Fi and Ethernet) analysis and video-streaming quality.

The scenarios are shown on subsequent figures. Scenario 1 (Figure 3) is composed of PLC adapters (HD Power-line Adapter with data rate $500 \mathrm{Mbps}$ ) and 2 PCs connected on each side.

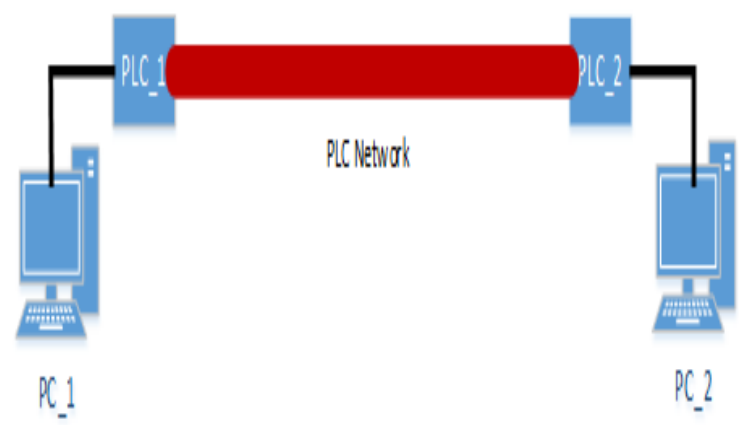

Fig.3. Scenario 1

Scenario 2 is composed from two links whereas scenario 3 is composed from a mixed network. The latest scenario is the most complicated compared to others. The size of video-streaming file is $700 \mathrm{MB}$ which is transmitted through the mixed network and Ethernet network, alternatively, with target to measure the quality of video in transient moments of different scenarios.

PLC adapters are used throughout all the scenarios, capable to transmit in data rates up to 500 Mbps and range distance up to $300 \mathrm{~m}$ for in-home environments. 


\subsection{Bandwidth, Jitter, Latency Measurements and Quality of Video-streaming}

Scenario 1 illustrates an example where PLC_1 (transmitter) transmits a video-streaming to PC_2 (receiver) through the Ethernet and PLC Network. The primary objective of this scenario is to measure the video quality at PC_2 for the data rates 10, 100 and 1000 Mbps. PLC adapters have capability of 500 Mbps data rates, but both of PLCs are equipped with $100 \mathrm{Mbps}$ NICs whereas $1000 \mathrm{Mbps}$ NICs are not used. It is obvious that in case of $10 \mathrm{Mbps}$ data rate the video quality transmitted via UDP on port 1234

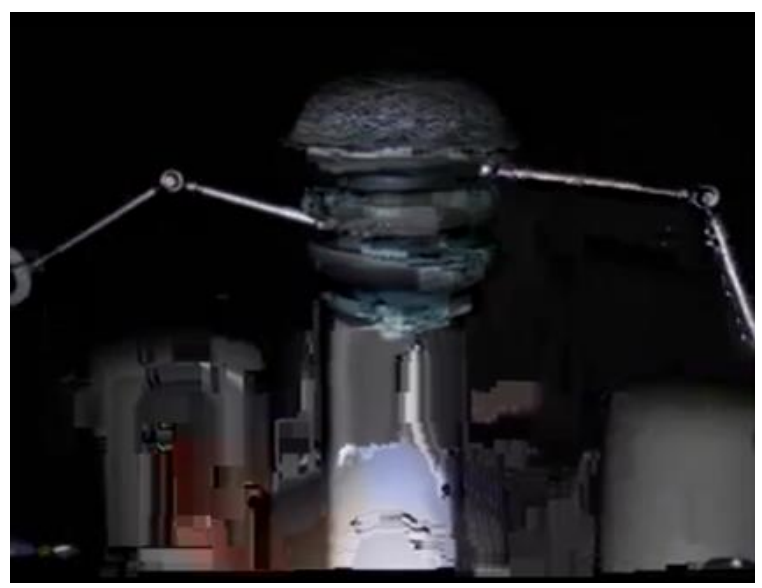

(User Datagram Protocol) has not changed. In the second test the data rate is increased to $100 \mathrm{Mbps}$, the incoming rates with value $96.5 \mathrm{Mbps}$ at PC_2 were recorded using Iptraf tool [7]. In this case quality of video is qualitatively degraded as it is shown on Figure 4. From this scenario it is understood that even when PLC adapters have a data rate of 500 Mbps, using cat5e Ethernet cables, it is not able to utilize the capacity of PLCs because of autonegotiation mechanism. This mechanism offers the data rates limited on 10/100/1000 Mbps as Ethernet standard does not support other data rates. The scenario 1 shows such a problematic solution in these situations.

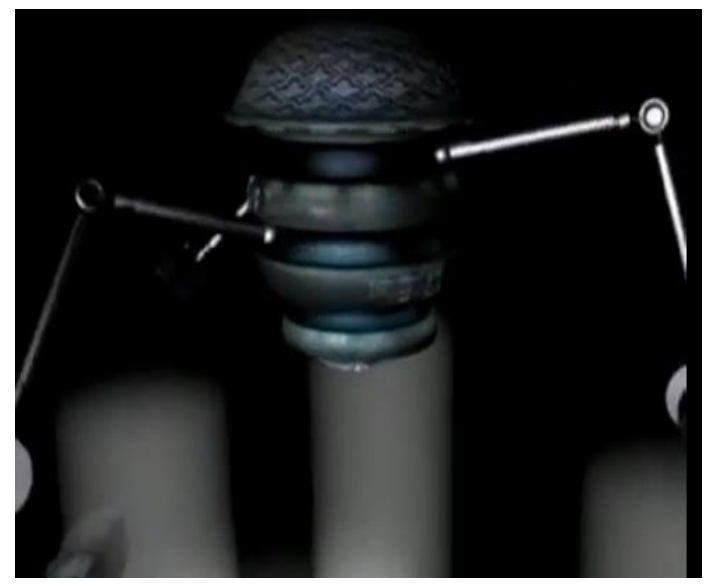

Fig.4. The degradation of video quality for $100 \mathrm{Mbps}$ data rate.

Scenario 2 as depicted on Figure 5 illustrates a different case where the PLC Network is used as a redundant link. On the interfaces of routers is activated the OSPF (Open Shortest Path First) protocol. In this test is forced an Ethernet link dropping down in order to measure the video quality at receiver PC as well as transient period. In case of Ethernet link goes down, it is observed a delay of up to $4 \mathrm{~s}$ for the video to turn into acceptable quality.

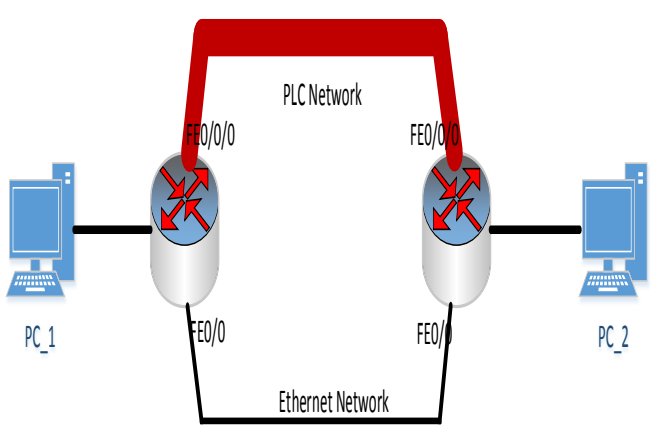

Fig.5. Scenario 2
Figure 6 shows the video quality at receiver side, whereas on right hand side of this one is shown the original image. This is done for comparison reasons where original image is used as a reference of comparison.

From the above figure we are also able to observe the time when the link is on switching phase from Ethernet to PLC link.

The load-sharing is such a mechanism that provides packet balancing which results in utilizing two links (Ethernet and PLC in our case). This is another test where on the router from left hand side the load-sharing per packet is enabled. On both sides (transmitter and receiver) are running the Jperf through which it has been able to measure jitter. 

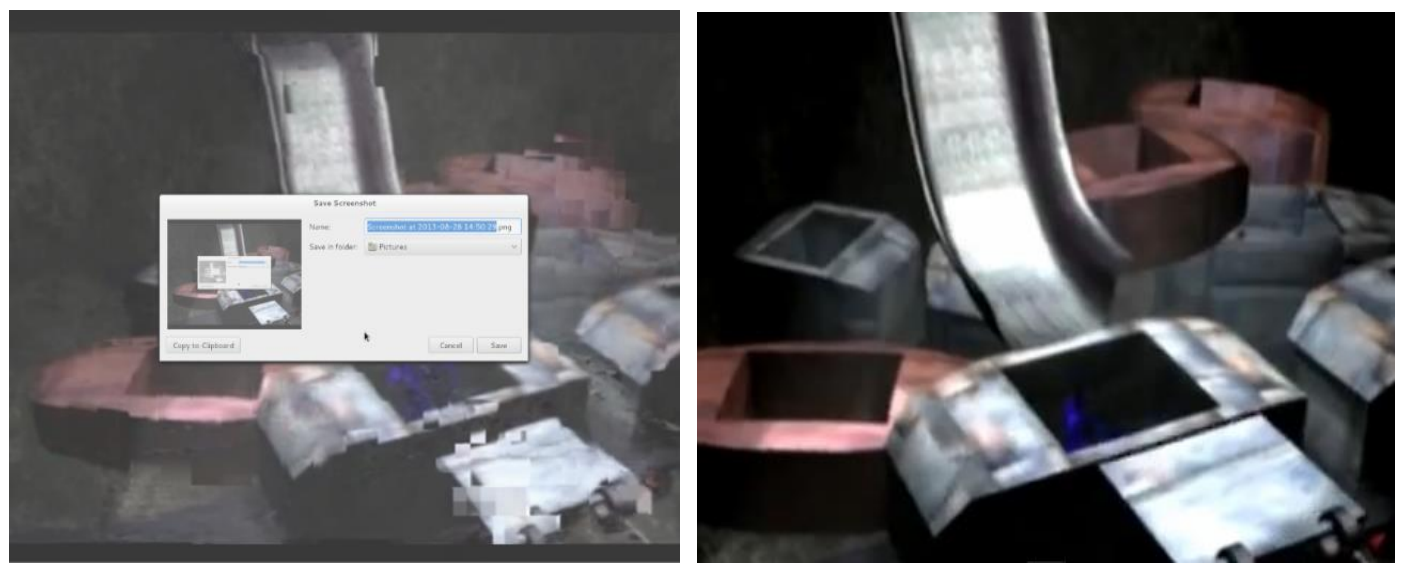

Fig.6. The degradation of video quality at the moments when link (Ethernet) goes down

Figure 7 shows the jitter results measured for the last $30 \mathrm{~s}$ (transmit interval is $60 \mathrm{~s}$ with 5 parallel streams). UDP is used with $10 \mathrm{MBps}$ throughput, 41 $\mathrm{kB}$ buffer size and $32 \mathrm{kB}$ packet size. Jitter results show that for this period the maximum value of jitter is $1.25 \mathrm{~ms}$. The real-time applications are sensitive to latency and jitter so a latency of more than $50 \mathrm{~ms}$ should be considered for different services.

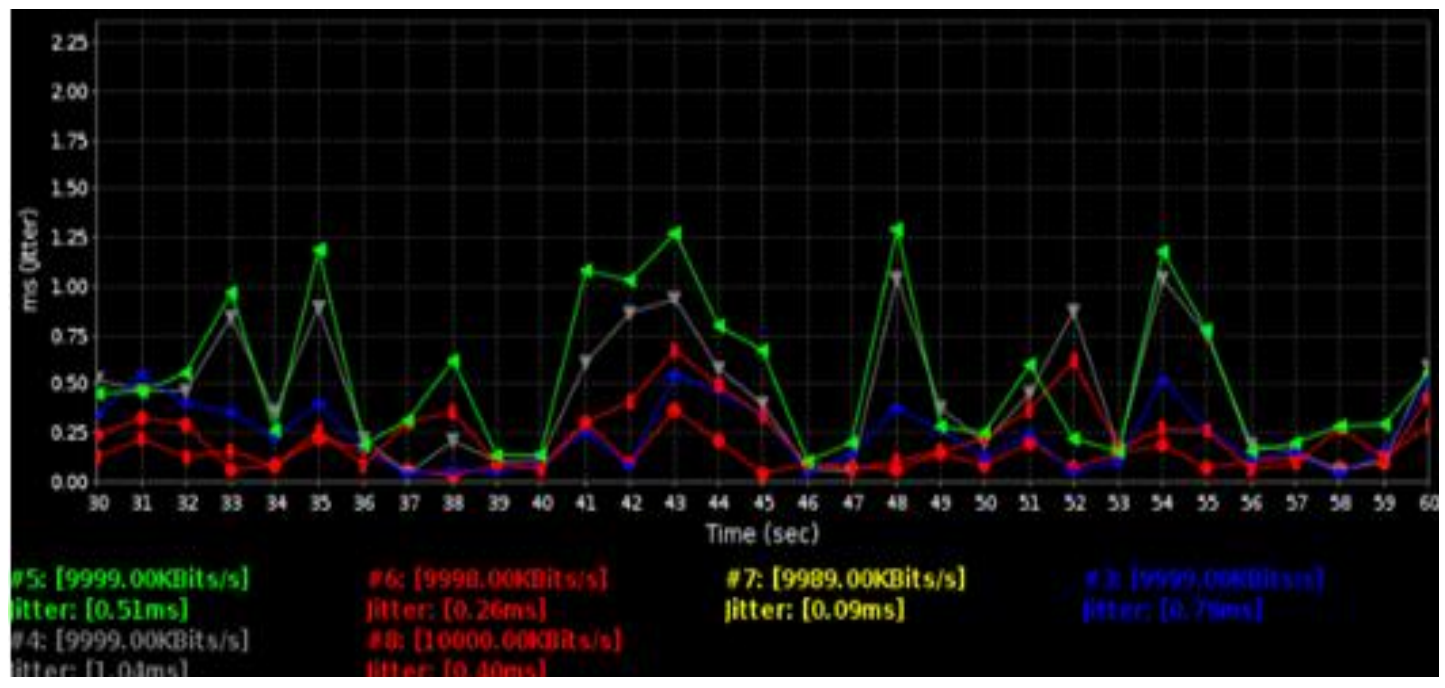

Fig.7. Jitter values for load-sharing per packet

Scenario 3 is more complicated in comparison to other scenarios because the network topology consists of two links (Figure 8) where the upper link is a combination of three technologies, Ethernet,

Wi-Fi and PLC.

After configuring the load-sharing per packet on router the traffic is divided, such that one packet goes via upper link (PLC and $\mathrm{Wi}-\mathrm{Fi}$ ) and the next one goes via lower link (Ethernet only).

A Wireshark capture is run on both sides of network topology (PC_1 and PC_2) [8]. Packets sent from transmitter to receiver are different in order.

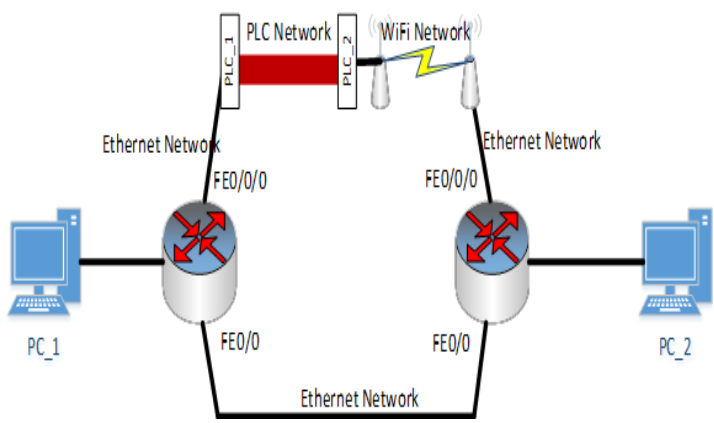

Fig. 8 Scenario 3

The load-sharing per packet affected MPEG packets (Figure 9) where is observed that a few packets to be lost. Therefore, in the situations of packet-sharing enabled the video quality may be degraded. 

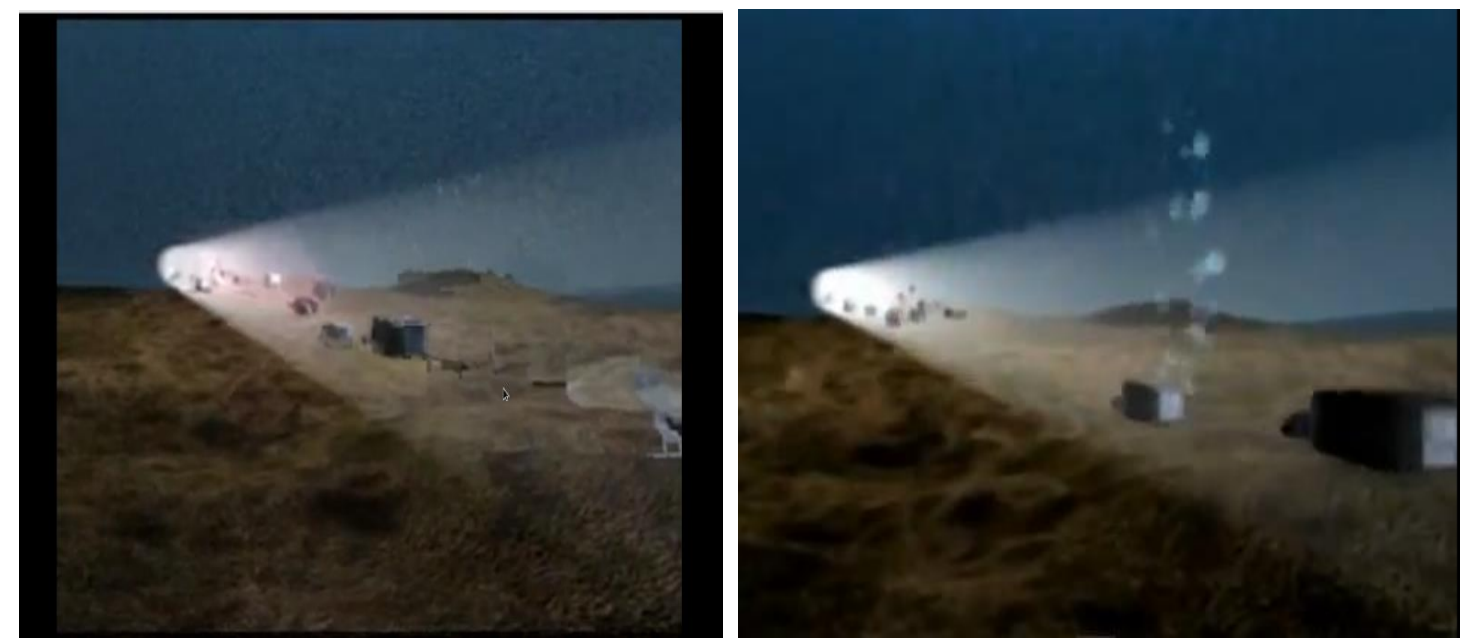

Fig.9. Video quality at receiver side (left image) in case of load-sharing per packet enabled

If the quality of the received video is compared to the original image captured at the transmitter side there are differences at the certain moments. However, this quality is acceptable because these degradations are appeared only in short intervals and non repeatable. Therefore, in these situations although is utilized the upper and lower capacity of links and also the packet-sharing is enabled this is not any more excellent video quality guarantee. Jperf is run similarly to scenario 2, on PC_1 and PC_2. Figure 10 shows the jitter measurements for 5 parallel streams. The testing interval is $60 \mathrm{~s}$. regarding to the jitter results observed, the value of jitter is significantly higher than the same results from scenario 2 . The jitter and also latency are increased due to $\mathrm{Wi}-\mathrm{Fi}$ and PLC networks.

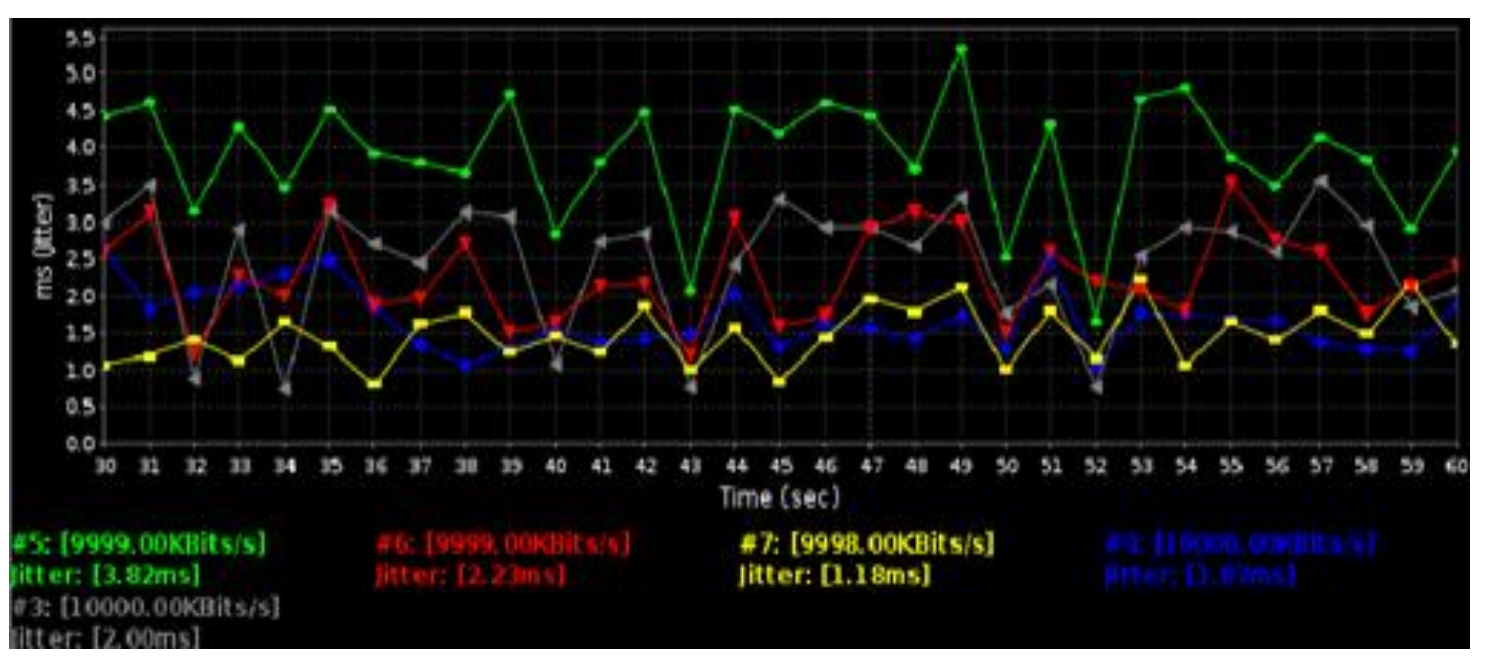

Fig.10. Jitter values for scenario 3 load-sharing per packet

Below are tabulated values for three cases related to the jitter and latency parameters about all mentioned technology combinations. It is interesting to observe the latency of packets for different cases. The mean values of latency are derived from 34 packets measured from ping test. Figure 11 illustrates the latency for every packet including cases of technology combinations.

If we relate Figure 11 and results from Table I (mean value of latency), it is able to observe the whole interval of packets whereby the combination of $\mathrm{Wi}$ $\mathrm{Fi}+$ Ethernet+PLC contains the largest values of latency.
TABLE I

JITTER AND LATENCY VALUES FOR DIFFERENT CASES JITTER AND LATENCY VALUES FOR DIFFERENT CASES.

\begin{tabular}{c|c|c|c}
\multirow{2}{*}{$\begin{array}{c}\text { Measured } \\
\text { values }\end{array}$} & \multicolumn{3}{|c}{ Load-sharing per packet enabled } \\
\cline { 2 - 4 } & $\begin{array}{c}\text { Wi-Fi } \\
\text { +Ethern } \\
\text { et +PLC }\end{array}$ & $\begin{array}{c}\text { WiFi+Eth } \\
\text { ernet }\end{array}$ & $\begin{array}{c}\text { Ethernet } \\
\text { + PLC }\end{array}$ \\
\hline $\begin{array}{c}\text { Max. } \\
\text { value of } \\
\text { jitter [ms] }\end{array}$ & 5.5 & 3.5 & 1.25 \\
\hline $\begin{array}{c}\text { Mean } \\
\text { value of } \\
\text { latency } \\
\text { [ms] }\end{array}$ & 5.3235 & 1.4412 & 3.0645 \\
\hline
\end{tabular}




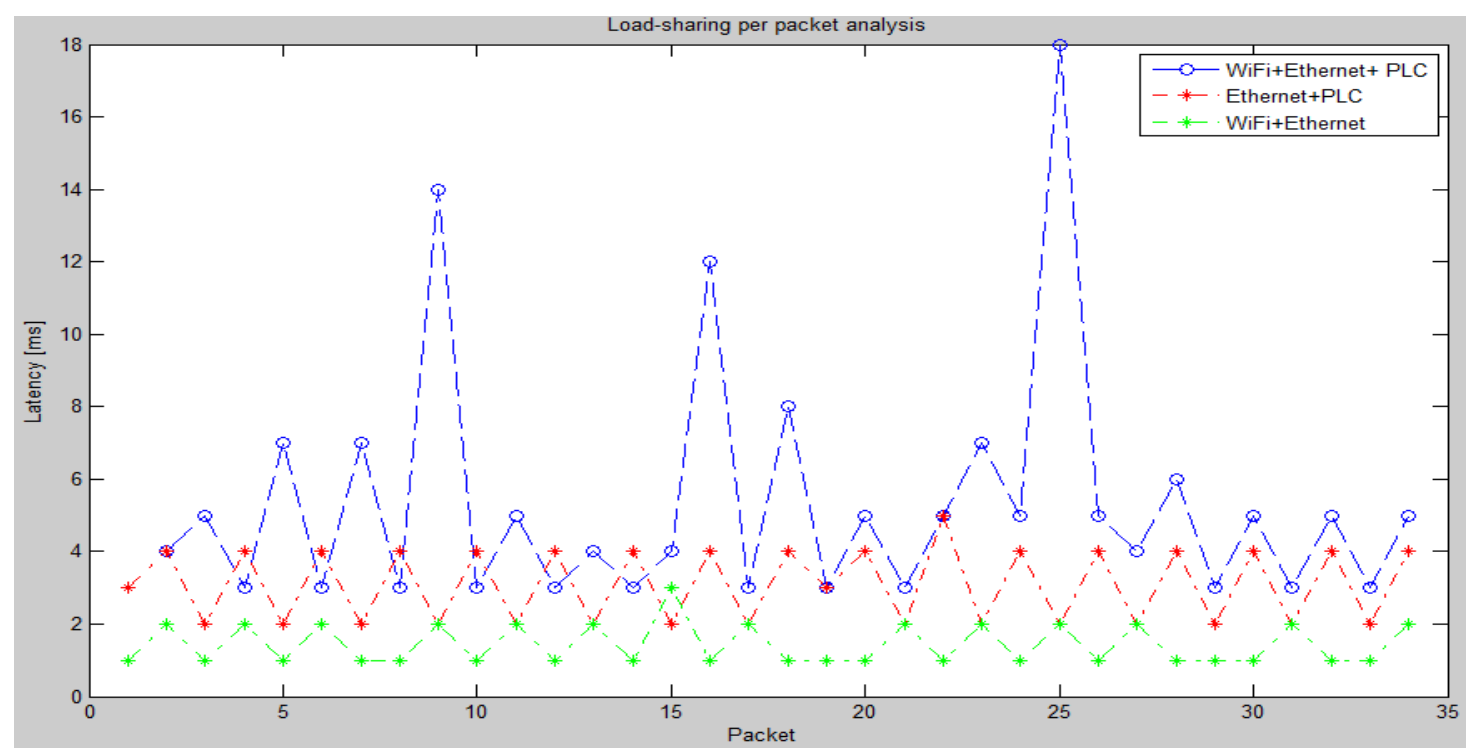

Fig.11. Latency values for 34 packets with various combination of technologies

\section{CONCLUSION}

Apart from home use the PLC technology apparently due to its good bandwidth characteristics there may be more alternatives where this technology may penetrate. The environments such as: Mining industry for monitoring purposes or Urgent cases whenever there is an already existent electrical infrastructure.

The channel transfer function is very important, so the results presented in this paper show a problematic bandwidth from 7-15 MHz. The data transmitted should be under control in order to make sure that the power noise level is not being lower than $-90 \mathrm{~dB}$. Also differences in distance range between outlets from different distribution boxes may cause changes in channel transfer function more than outlets positioned within the same distribution box. In the situations where the network topology is complicated there are presented more oscillations on channel transfer function.

In case of using PLC as a redundant system, this operates very well regardless some seconds of video degradation. This video degradation occurs to be at the moments when secondary link gets recovered from primary link. In case of using the load-sharing per packet mechanism the situation may change somehow. Although the idea of using the both links is desirable, there is needed more attention when a few technologies are used in combination, as latency and jitter are fundamental parameters for real-time applications.

\section{REFERENCES}

[1] Marrocco G, Statovci D, and Trautmann S. A PLC Broadband Channel Simulator for Indoor Communications, International Symposium on Power Line Communications and Its Applications (ISPLC), pp. 321-326, March 2013.

[2] FTW PLC Simulator, Online Available. http://plc.ftw.at.

[3] Golden P, Dedieu H, and Jacobsen K. S. Fundamentals of DSL Technology, 1st ed. Auerbach Publications, July 2004.
[4] IEEE Standard for Broadband over Powerline Networks. Medium access Control and Physical Layer Specifications, IEEE1901, pp. 11586, December 2010

[5] Andrea M. T, Fabio V. IEEE, Bottom-Up Statistical PLC Channel Modeling Part II: Inferring the Statistics, vol. 25, no. 4, pp. 23562363, October 2010.

[6] Canete F.J, Díez L, Cortes J.A, Entrambasaguas J.T. Broadband modeling of indoor power-line channels, vol. 48, no. 1, pp. 175-183, February 2002.

[7] IPTraf 3.0.0, National Center for Supercomputing, University of Illinois at urbana-Champaign: http://dast.nlanr.net/Projects/Iperf/, September, 2005.

[8] Wireshark network protocol analyzer, Wireshark $®$ is available under the GNU General Public License version 2: https://www.wireshark.org/.

\section{BIOGRAPHIES}

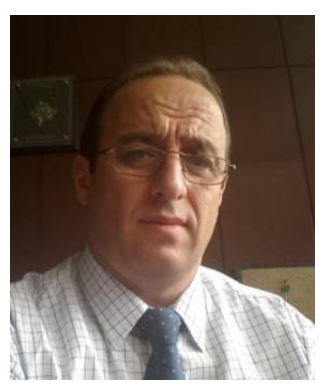

ENVER HAMITI received Dipl. Ing. degree in Electronics and Telecommunications (1990), Mr.sc and $\mathrm{Ph} . \mathrm{D}$. in the field of Telecommunications, in 1999 respectively 2006 from the University of Prishtina, Faculty of Electrical and Computer Engineering. He was appointed as Associate Professor in the department of Telecommunications at the University of Prishtina, Faculty of Electrical and Computer Engineering, in 2010. His research interests cover the wide spectrum of wireless communications. In a particular he is interested in: antenna engineering, analytical and computational electromagnetics, microwave systems, modeling techniques for simulation of electromagnetic waves, electromagnetic field propagations and safe wireless and cellular communications. He has authored or co-authored many conference and journal papers. 


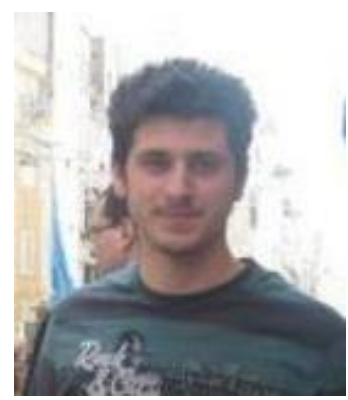

TAULANT BERISHA received his BS and Msc degree in Electrical Engineering from the University of Prishtina, Faculty of Electrical and Computer Engineering in 2013. His research interests are in the broad area of wireless communications and networking.

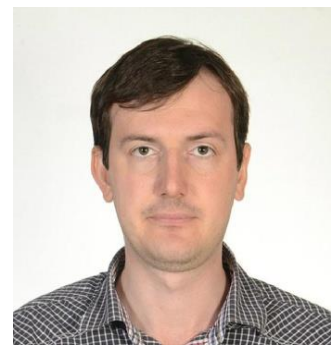

FATOS PECI received his BS degree in Electrical Engineering from the University of Prishtina, Faculty of Electrical and Computer Engineering in 2007, while. Master's Science deegre in Networking and Telecommunication Engineering at University of Pittsburgh. His research interests are in the broad area of wireless communications and networking, data structures, database systems. 